\title{
Reply to commentary of Carr and Kettle, the challenge of quantifying susceptibility to drought-related crisis
}

\author{
Joseph Alcamo $\cdot$ Lilibeth Acosta-Michlik • \\ Alexander Carius · Frank Eierdanz
}

Received: 30 March 2009/Accepted: 5 April 2009/Published online: 30 April 2009

(c) Springer-Verlag 2009

After reading Carr and Kettle's text, we have identified three main arguments:

1. The models make wrong basic assumptions ("... the selection of the state as the object of analysis runs contrary to extensive literature ...")

2. The models leave things out ("... we are not convinced that their model has captured other important determinants ...")

3. Pragmatically selecting indicators is a bad thing ("We are concerned that the continued legitimization of variables based on pragmatism will lead to an air of scientific legitimacy surrounding the exclusion of important data-limited indicators, and reinforce current data gathering foci.”)

With regards to the first argument, we believe that the scientific community is far from having consensus about the main factors or processes determining the susceptibility/vulnerability of society to drought or any extreme climate events for that matter. These issues are argued about in the literature with the same ferocity as a rugby match. The point is that there is no consensus about the correct basic assumptions for a vulnerability model. Carr

J. Alcamo $(\square)$

Center for Environmental Systems Research,

University of Kassel, Kassel, Germany

e-mail: alcamo@usf.uni-kassel.de

L. Acosta-Michlik

University of Louvain, Louvain, Belgium

A. Carius

Adephi Research, Berlin, Germany

F. Eierdanz

Institute for Technology and Work, Kaiserslautern, Germany and Kettle provide good arguments for including certain assumptions, but we think the texts of Tänzler et al. and Acosta-Michlik et al. provide equally good justification for making alternative assumptions.

With regards to the second argument, that certain factors were left out of the model, we would argue that no model is so complete that it does not leave some factors out; the fact of the matter is that models are, per definition, simplified versions of reality, and do not include everything.

And finally, with regards to the third argument, we sympathize with the Commentators' points about selecting indicators pragmatically; but, we doubt whether we can be any less pragmatic in selecting data for our models than we are about choosing our route to work; you know, we may not like the crowded trains, but we have to get to the workplace. We may not like the limitations of current data, but unless we use them to build our models, we will not be able to understand where the data deficiencies lie. Put another way, building models based upon best available data stimulates a helpful discussion amongst the scientific community as to which data need to be collected next in order to improve the model.

In general, we find that the Commentary of Carr and Kettle misses an important point of the Special Issue, which is embodied in its title "Quantifying vulnerability to drought from different disciplinary perspectives" (underscore added). The fact of the matter is that there are now several different perspectives on vulnerability, and it is not possible to say which is more valid than the other. It is our opinion that the scientific community should embrace these differences and concentrate on learning why these differences exist rather than arguing why one model is better than another.

In conclusion, we encourage researchers to develop and test internally consistent models of susceptibility/vulnerability, despite the fact that there will always be other 
theories to explain vulnerability, despite the fact that some factors will be left out, and despite the fact that some degree of pragmatism is needed to fill these models with data.

These models are urgently needed in order to:

- help identify where vulnerability in the world is of special concern

- help determine which communities, social groups, or regions are more vulnerable than others
- help to explain why different academic disciplines propose different explanations of vulnerability

- help link the social and biophysical aspects of climate and its impacts on society.

The accent here is on "help" because not only modelling, but also practical on-the-ground experience and expert knowledge are, of course, required for these tasks. 